\author{
Journal homepage:http://www.interscience.org.uk \\ DOI:10.18535/ijahm/v8i1.03 \\ Impact Factor: 4.415
}

\title{
Protective Effect Of Aabresham (Bombyx Mori) Against Atherosclerosis
}

\author{
${ }^{1}$ Aliya Parveen, ${ }^{2}$ Dr Zarnigar \\ ${ }^{1}$ Assistant Professor,Department of Community Medicine,RUMCHRC,Jaipur-Rajasthan \\ ${ }^{2}$ Lecturer, Department of Community Medicine,NIUM,Bangaluru,Karnataka
}

Objectives: To assess the efficacy of Aabresham (Bombyx mori) for the prevention of atherosclerosis.

\begin{abstract}
Methods: Randomized single blind standard control risk factor trial was conducted in National Institute of Unani Medicine Hospital, Bangalore from March 2015 to March 2016. Fifty patients with risks of atherosclerosis were selected and randomly assigned into two groups 30 in test group and 20 in control group. Test group was treated with the decoction of Aabresham (Bombyx mori), six grams in the morning empty stomach for two months and control group was treated with Atorvastatin, ten mg, in the evening for the same duration. The response was evaluated by the assessing the subjective and objective parameters. The result was analyzed statistically by using Fisher Exact test, ChiSquare test and Student' $t$ ' test (Unpaired \& Paired).
\end{abstract}

Results: Test group showed significant reduction in breathlessness, palpitation, headache, blood pressure, serum cholesterol, Low density lipoprotein (LDL) \& MDA (Malonedialdehyde)

concentration $(\mathrm{p}<0.01)$ but chest pain, serum triglyceride, haematocrit and HDL(High density lipoprotein) values were found statistically insignificant in both groups. Overall, improvement was observed in test group except blood urea.

Conclusion: Aabresham(Bombyx mori shown to have an astounding potential in the prevention of atherosclerosis. It's anti-oxidant, anti inflammatory, platelet stabilizing and anti- arrhythmic effect plays an unparallel role in the inhibition of development \& progression of atherosclerosis. Integration with regimental therapies and dietary modification will potentiate its efficacy.

Keywords: Atherosclerosis, Marz-e-majari, Sudda, Aabresham

\section{Introduction:}

The history reveals that the story of Atherosclerosis was first narrated by Leonardo da Vinci, 1400 years ago as thickening of tunics, which continues till the identification of oxidized Low density lipoprotein(LDL) by Daniel Steinberg and his group in 1980s. ${ }^{1}$ Atherosclerosis is best defined as a condition where arteries become narrowed and hardened due to the formation of plaque around the arterial wall. The disease disrupts the blood flow to the vital organs posing serious cardiovascular complications like Myocardial infarction, Stroke or sudden death. ${ }^{2}$ Cardiovascular diseases are the leading non-communicable diseases; nearly half of the 36 million deaths due to non-communicable diseases contributed to Cardiovascular diseases(CVDs) and 10\% of global disease burden is attributed to CVDs. ${ }^{3}$ In India, the mortality has increased from 2.25 million (2010) to 2.94 million (2015). ${ }^{4}$ In the vasculature, an increased Reactive oxygen species(ROS) defines the pathobiological state of oxidative stress. ROS play an integral, mechanistic role in all aspects of atherogenesis and modify the factors that govern the vascular redox state, increasing the risk for the development of atherothrombotic cardiovascular disease. ${ }^{5}$ It has been seen that over last two decades mortality due to CVDs has declined in developed countries due to a combine approach of prevention \& control against dyslipidemia. A number of hypolipidemic drugs have been already introduced in the modern system of medicine. Among them, Statins have gained the popularity from years. But their adverse effects and high cost exasperates the condition.

Unani system of medicine described atherosclerosis as a type of Marz-e- majari (disease of vessels) in which sharain (arteries) become narrow and obstructed by akhlat-e-ghaleeza (vicid humours/sauda) and akhlat-eluzuja (sticky humours/phlegm). ${ }^{6}$ Unani concept gave a radical knowledge regarding the modern day concept 
of oxidative stress to some extent and Unani drugs have demonstrated positive effects in modifying risk factors for CVDs. Some of the Unani drugs have shown hypolipidemic, anti-atherogenic, antioxidant and antiinflammatory effects. One of them is Aabresham (Bombyx mori), which is mentioned as Mufarrah wa Muqawwie Qalb (Cardiotonic), Mufattite sudud (Deobsruent) Mulattif (Demulcent), Munaqqie balgham wa sauda (Purgative) and Musaffi dam(Blood purifier) in classical text of Unani medicine. ${ }^{7,8.9 .10,11}$ Thus, the present study is conducted to evaluate the protective effect of Aabresham (Bombyx mori) against atherosclerosis using modern day tools. If the study found to succeed in its objective, it will help to fill the gap in the knowledge to some extent.

\section{Subjects and Methods:}

Study was conducted after obtaining the ethical clearance by the Institutional Ethical Committee of National Institute of Unani Medicine, Bangalore, India (Reg. No. 07_U006_51360).

Inclusion Criteria: Patients of either sex, age group 35-60 years, B.P $\geq 140 / 90 \mathrm{~mm} \mathrm{Hg}$ or on antihypertensive medication, Serum triglyceride $(150-499 \mathrm{mg} / \mathrm{dl})$ and Low density lipoprotein $(100-189 \mathrm{mg} / \mathrm{dl}){ }^{12}$

Exclusion Criteria: Patients below the age of 35 years and above 60 years, bleeding disorders and uncontrolled Blood Pressure.

Selection of subjects: Patients were inquired about their demographic profile and socioeconomic status. For socioeconomic status, Kuppuswamy's socioeconomic scale was used (Modified 2014, Jan). ${ }^{13}$ All the patients were interrogated about their chief complaints especially regarding palpitation, headache, dizziness, breathlessness, chest pain, fatigue, insomnia, nervousness, dyspnoea \& epistaxis with duration. Detailed history of any organic disease (especially cardiovascular diseases and Diabetes), family history, personal, social, any treatment and gynaecological or obstetrical history was also taken. General physical examination and a careful systemic examination of cardiovascular system, respiratory system, gastrointestinal system, renal system and nervous system were carried out to look for any finding or other serious illness. Any positive finding or regression or progression of symptoms was recorded in the Case record form designed in accordance to the objectives of the study.

Investigations: Certain investigations were carried out to exclude the patients, to assess the efficacy and to establish the safety of test drug. Haemoglobin\%, Total Leucocytes count, Differential Leucocyte count, Bleeding time, Clotting time, Erythrocyte Sedimentation Rate, Renal function test, Liver Function test, Lipid peroxidation test (MDA concentration assessment by Ohkawa et al 1979), lipid profile, random blood sugar and haematocrit were done in every case before and after intervention.

Method of preparation and mode of administration of test and control drugs

Test drug Aabresham (Bombyx mori) and control drug Atorvastatin were provided by National Institute of Unani Medicine. Proper identification of the drug was done by chief pharmacist, National Institute of Unani Medicine, to ensure their originality and authenticity. The drug was cleaned to remove any unwanted material and impurities. Test group was treated with six gm of Aabresham (Bombyx mori) in the morning, empty stomach for two months as decoction. While Control group was treated with Atorvastatin ten mg orally in the evening for the same period of time.

Follow up: Patients were kept under strict observation and advised to come every $15^{\text {th }}$ day to the hospital and were asked about the progression or regression in their symptoms, and subjected to assess the clinical findings. Concomitant treatment was not allowed during the study in both groups.

Efficacy assessment: The assessment of the efficacy in both the groups was based on objective and subjective parameters. Assessment of objective parameters like blood pressure measurement was done on every $15^{\text {th }}$ day while, lipid profile, haematocrit and MDA assessment was done before and after intervention. Subjective parameters like palpitation, breathlessness, headache and chest pain were assessed by an arbitrary grading, as these parameters differ in severity from patient to patient. Before starting treatment, baseline observations were recorded in the case report form and any worsening or improvement in the parameters was noted down at every visit of follow up till the end of the treatment. After 60 days of the treatment, values of different parameters were analyzed and compared statistically.

Safety assessment: Clinical assessment at every visit of follow up, Haematological assessment (before and after the treatment) -Haemoglobin \%, Total Leucocytes count, Differential Leucocytes count, Bleeding time, Clotting time, Erythrocyte Sedimentation Rate, Biochemical assessment (before and after the 
treatment) - Liver Function test (SGOT, SGPT), Renal function test (Blood Urea, Serum Creatinine) and Random blood sugar.

Statistical analysis: Statistical analysis was carried out by using software namely SAS 9.2, SPSS 15.0, Stata 10.1, Med Calc 9.0.1, Systat 12.0 and R environment ver.2.11.1 Significance is assessed at $5 \%$ level of significance. Student t, test has been used (two tailed, independent for Inter group analysis and two tailed, dependent within each group). Chi-square/ Fisher Exact test has been used on categorical scale between two or more groups.

\section{Results \&Discussion:}

Maximum numbers of subjects studied were females under the age group of 51-60 years. Most of the females in this age group had attained menopause. Estrogen plays a protective role against atherosclerosis. The risk of development of CVDs increases 3.4 times after menopause. These observations are similar to that of J. C. Witteman et al (1989). ${ }^{14}$ Nearly $78 \%$ (39) of study subjects were non vegetarian (mixed diet) and $22 \%$ (11) were vegetarian. This study corresponds to the study of Manish Verma and group (2015) ${ }^{15}$, whose results specified that the mean total cholesterol levels in their study was significantly increased in non-vegetarians that raised the risk for overweight and obesity. Vegetarian diet is comprised of fibres which increase bile-acid binding, faecal sterol excretion, and fermentation of soluble fibres that produce shortchain fatty acids which inhibit hepatic cholesterol synthesis. As the study population is mainly comprised of females therefore, $86 \%$ (43) subjects found to be non smokers, whereas 14\% (7) were smokers. Cigarette smoking may increase the risk of CVD by promoting atherosclerosis progression and other triggering factors like changing in homeostasis. Both active smoking and exposure to smoke are associated with the progression of atherosclerosis. The outcome of our observation are quite similar to that of George Howard et al $(1998){ }^{16}$ where, although most of the participants were females yet developed atherosclerosis as they were passively exposed to smoking. 36\%(18) of subjects virtually belonged to upper lower class (IV), $24 \%$ (12) to upper middle class(II), 20\%(10) from lower middle class (III), 16\%(8) upper class I and 4\% (two) from lower class(V). CVD risk factors among the urban poor and middle class are rapidly increasing in India. The probable reason for this is an "aspiration effect" which has behavioural consequences among the rich people in India. The aspiration to emulate the western society leads to improved awareness of the preventive measures to tackle the raised CVD risk in these groups. By contrast, higher levels of tobacco use, obesity or overweight and hypertension are now associated with lower levels of education and income in India. Our study coincides with the results of P. Jeemon and K. S. Reddy. ${ }^{17}$ Almost $12 \%$ (24) subjects under study were found to had a positive family history of cardiovascular diseases and the remaining 72\% (36) subjects had no family history of CVD. Our study demonstrated the same results as by Arvind $\mathrm{K}$ et al ${ }^{18}$ suggested that family history may have a "dose-dependent" effect, where individuals with more robust family histories of CHD are at greater risk of developing CVDs. It had a significant increase in incidence and progression of coronary artery calcification. Our study comprised of 32\% (16) pre-obese subjects, $24 \%$ (12) obese class I, $22 \%$ (11) obese class II, $8 \%$ (4) obese class III. Along with this $76 \%$ (38) of subjects spend a sedentary life style, $16 \%$ (8) engaged in moderate physical activity and $8 \%$ (4) mild physical activity. Sedentary lifestyle increases all causes of mortality, double the risk of cardiovascular diseases, obesity, high blood pressure \& lipid disorders. An increased risk of death, CVD, Coronary heart disease and ischemic stroke was observed in pre obese subjects (BMI> 24.9), studied by Yu Chen et al (2013) which resembles with the observations made by us. ${ }^{19,20}$ (Table No.1)

Table No.1: Demographic profile of patients

\begin{tabular}{|l|l|l|l|l|l|}
\hline \multirow{2}{*}{ Parameters } & \multicolumn{2}{|l|}{ Test Group } & \multicolumn{2}{l}{ Control Group } & \multirow{2}{*}{ Total (\%) } \\
\cline { 1 - 5 } Age (years) & \multicolumn{2}{|l}{ Males } & \\
\cline { 2 - 5 } & Females & Males & Females & Mal \\
\hline $31-40$ & $6(20 \%)$ & $4(13.3 \%)$ & $3(15 \%)$ & $1(5 \%)$ & $14(28 \%)$ \\
\hline $41-50$ & $6(20 \%)$ & $2(6.6 \%)$ & $5(25 \%)$ & $2(10 \%)$ & $15(30 \%)$ \\
\hline $51-60$ & $8(26.7 \%)$ & $4(13.33 \%)$ & $7(35 \%)$ & $2(10 \%)$ & $21(42 \%)$ \\
\hline
\end{tabular}


Aliya Parveen, International Journal of Ayurvedic \& Herbal Medicine 8 (1) Jan.-Feb. 2017 (3069-3078)

\begin{tabular}{|c|c|c|c|c|c|c|}
\hline \multicolumn{2}{|l|}{ Total } & $20(66.7 \%)$ & $10(33.3 \%)$ & $15(75 \%)$ & $5(25 \%)$ & $50(100 \%)$ \\
\hline \multicolumn{7}{|l|}{ Diet } \\
\hline \multicolumn{2}{|l|}{ Mixed } & \multicolumn{2}{|l|}{$25(83.3 \%)$} & \multicolumn{2}{|l|}{$14(70 \%)$} & $39(78 \%)$ \\
\hline \multicolumn{2}{|l|}{ Veg } & \multicolumn{2}{|l|}{$5(16.7 \%)$} & \multicolumn{2}{|l|}{$6(30 \%)$} & $11(22 \%)$ \\
\hline \multicolumn{7}{|c|}{ H/O Smoking } \\
\hline \multicolumn{2}{|c|}{ Non Smoking } & \multicolumn{2}{|l|}{$24(80 \%)$} & \multicolumn{2}{|l|}{$19(95 \%)$} & $43(86 \%)$ \\
\hline \multicolumn{4}{|c|}{\begin{tabular}{l|l|} 
Smoking & $6(20 \%)$ \\
\end{tabular}} & \multicolumn{2}{|l|}{$1(5 \%)$} & $7(14 \%)$ \\
\hline \multicolumn{7}{|c|}{ Socio Economic Status } \\
\hline $6(20 \%)$ & \multicolumn{3}{|c|}{$6(20 \%)$} & \multicolumn{2}{|l|}{$2(10 \%)$} & $8(16 \%)$ \\
\hline II & \multicolumn{3}{|c|}{$7(23.3 \%)$} & $5(25 \%)$ & & $12(24 \%)$ \\
\hline III & \multicolumn{3}{|c|}{$4(13.3 \%)$} & $6(30 \%)$ & & $10(20 \%)$ \\
\hline IV & \multicolumn{3}{|c|}{$11(36.7 \%)$} & $7(35 \%)$ & & $18(36 \%)$ \\
\hline $\mathbf{V}$ & \multicolumn{3}{|c|}{$2(6.7 \%)$} & $0(0 \%)$ & & $2(4 \%)$ \\
\hline \multicolumn{7}{|c|}{ F/H of CVD } \\
\hline Absent & \multicolumn{3}{|c|}{$22(73.3 \%)$} & \multicolumn{2}{|l|}{$14(70 \%)$} & $36(72 \%)$ \\
\hline Present & \multicolumn{3}{|c|}{$8(26.7 \%)$} & \multicolumn{2}{|l|}{$4(20 \%)$} & $12(24 \%)$ \\
\hline \multicolumn{7}{|c|}{ Distribution of BMI" } \\
\hline \multicolumn{2}{|c|}{ Under Weight } & \multicolumn{2}{|c|}{$5(16.7 \%)$} & $0(0 \%)$ & & $5(10 \%)$ \\
\hline Normal & & $2(6.7 \%$ & & $0(0 \%)$ & & $2(4 \%)$ \\
\hline Pre Obes & & $9(30 \%)$ & & $7(35 \%)$ & & $16(32 \%)$ \\
\hline Obese cla & & $8(26.7 c$ & & $4(20 \%)$ & & $12(24 \%)$ \\
\hline Obese cla & s II & $6(20 \%)$ & & $5(25 \%)$ & & $11(22 \%)$ \\
\hline Obese cla & s III & $0(0 \%)$ & & $4(20 \%)$ & & $4(8 \%)$ \\
\hline Physical & ctiv & & & & & \\
\hline Sedentary & & $23(76.7$ & & $15(75 \%)$ & & $38(76 \%)$ \\
\hline Moderate & & $5\left(16.7^{c}\right.$ & & $3(15 \%)$ & & $8(16 \%)$ \\
\hline Mild & & $2(6.7 \%$ & & $2(10 \%)$ & & $4(8 \%)$ \\
\hline
\end{tabular}

*BMI $=$ Body mass index

While assessing the subjective parameters, our observations showed statistically a significant reduction in breathlessness, palpitation, headache and chest pain in both the groups, after intervention as compared to before intervention $(\mathrm{p}<0.001)$. Whereas clinically Test group showed highly significant reduction in breathlessness, palpitation, headache and chest pain on day $60^{\text {th, }}$ with respect to 0 day.(Fig No.1)

Fig No 1

Effect of drug on Breathlessness, Palpitation, Headache \&Chest pain in Atherosclerosis risk subjects

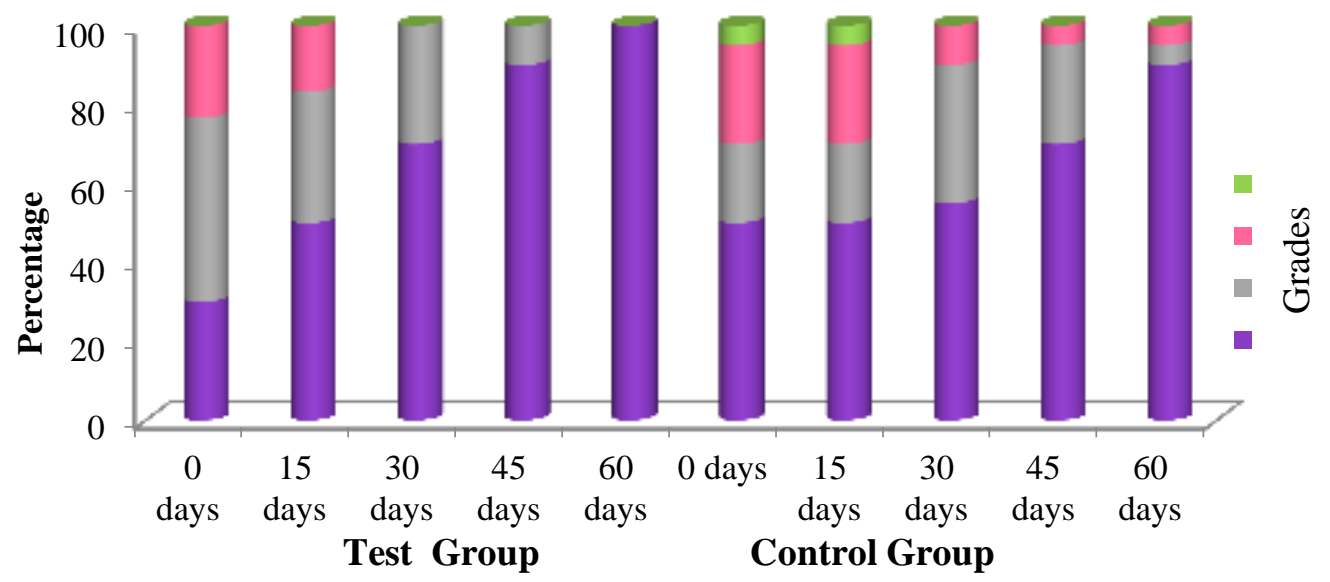


Table No.2

Effect of drug on BP, Cholesterol, Triglycerides, LDL \& MDA concentration in Atherosclerosis risk subjects (Mean \pm SEM)

\begin{tabular}{|c|c|c|c|}
\hline SBP (mm Hg) & Test Group & $\begin{array}{l}\text { Control } \\
\text { Group }\end{array}$ & $P$ value \\
\hline Before Treatment & $149.23 \pm 17.49$ & $141.70 \pm 11.18$ & $0.095+$ \\
\hline After Treatment & $123.50 \pm 10.32$ & $124.50 \pm 16.69$ & 0.794 \\
\hline Difference & 25.733 & 17.200 & 0.181 \\
\hline$P$ value & $<0.001 * *$ & $0.002 * *$ & - \\
\hline \multicolumn{4}{|l|}{ DBP (mm Hg) } \\
\hline Before Treatment & $93.97 \pm 6.11$ & $91.40 \pm 8.17$ & 0.210 \\
\hline After Treatment & $82.53 \pm 6.58$ & $84.45 \pm 6.31$ & 0.310 \\
\hline Difference & 11.433 & 6.950 & 0.127 \\
\hline$P$ value & $<0.001 * *$ & $0.009 * *$ & - \\
\hline \multicolumn{4}{|c|}{ Cholesterol (mg/dl) } \\
\hline Before Treatment & $215.87 \pm 30.46$ & $209.00 \pm 21.45$ & 0.387 \\
\hline After Treatment & $180.33 \pm 43.54$ & $168.30 \pm 34.91$ & 0.307 \\
\hline Difference & 35.533 & 40.700 & 0.646 \\
\hline P value & $<0.001 * *$ & $<0.001 * *$ & - \\
\hline \multicolumn{4}{|c|}{ Triglycerides (mg/dl) } \\
\hline Before Treatment & $186.00 \pm 90.93$ & $183.15 \pm 70.17$ & 0.906 \\
\hline After Treatment & $167.60 \pm 66.06$ & $157.55 \pm 36.55$ & 0.539 \\
\hline Difference & 18.400 & 25.600 & 0.741 \\
\hline$P$ value & 0.227 & $0.086+$ & - \\
\hline \multicolumn{4}{|l|}{ LDL (mg/dl) } \\
\hline Before Treatment & $133.97 \pm 21.59$ & $129.88 \pm 18.46$ & 0.491 \\
\hline After Treatment & $104.19 \pm 38.06$ & $93.08 \pm 30.60$ & 0.281 \\
\hline Difference & 29.787 & 36.800 & 0.499 \\
\hline$P$ value & $<0.001 * *$ & $<0.001 * *$ & - \\
\hline \multicolumn{4}{|l|}{ MDA (nmol/ml) } \\
\hline Before Treatment & $1.96 \pm 1.11$ & $1.72 \pm 0.92$ & 0.434 \\
\hline After Treatment & $0.29 \pm 0.16$ & $0.34 \pm 0.15$ & 0.307 \\
\hline Difference & 1.670 & 1.387 & 0.339 \\
\hline$P$ value & $<0.001 * *$ & $<0.001 * *$ & - \\
\hline
\end{tabular}

\section{Blood pressure}

In present study both the groups showed highly significant reduction in systolic and diastolic blood pressure after treatment as compared to before treatment with $\mathrm{p}<0.01$. The intra group comparison exhibited that Mean \pm SEM(Standard error of mean) score difference in test group for Systolic blood pressure(SBP) was 25.733 and for Diastolic blood pressure (DBP) was 11.433 as compared to control group which was 17.200 for SBP and 6.950 for DBP on day $60^{\text {th }}$ with respect to day 0. (Table No.2) The above observations showed that there is a significant reduction in systolic and diastolic blood pressure in test group with $\mathrm{p}<0.001$, as compared to control group after intervention with respect to before intervention.

\section{Serum cholesterol}

Clinically and statistically both the drugs reduced the total serum cholesterol level to a highly significantly level on day $60^{\text {th }}$ with respect to day $0, \mathrm{p}<0.001$. (Table No.2)

\section{Serum Triglycerides}

Clinically both the drugs were found to be almost equally effective in reducing serum triglycerides level with $\mathrm{p}=0.539$ after intervention. (Table No.2)

\section{Low density lipoprotein-Cholesterol (LDL-C)}


The intra and inter group comparison indicated that there is a marked significant reduction in LDL-C level in both the groups, $\mathrm{p}<0.001$ after intervention in comparison to baseline values. Therefore, both the drugs found to be equally effective in reducing LDL-C on day $60^{\text {th }}$ with respect to day 0.(Table No.2)

\section{High density lipoprotein-Cholesterol (HDL-C)}

The statistical assessment showed no difference in the increment of HDL-C after invention with $\mathrm{p}=0.719$ compared to baseline values. Hence, none of the drug is found to be statistically efficient in increasing HDLC.

\section{MDA (Malonedialdehyde) concentration}

The Mean \pm SEM MDA concentration difference in test group was found as 1.670 on day $60^{\text {th }}$ with respect to 0 day. The Mean \pm SEM MDA concentration difference in control group was 1.387 on $60^{\text {th }}$ day in comparison to day 0 . Statistically both the drugs imparted significant reduction in MDA concentration after intervention with respect to baseline values, $p<0.001$. (Table No.2)MDA is a marker of oxidative stress and in many studies; it showed highly significant relation with coronary artery diseases revealing the interrelation between the oxidative stress and atherosclerosis. The MDA, a carbonyl group produced during lipid peroxidation, is used widely in determining oxidative stress. Decrease in MDA concentration indicates decrease in lipid peroxidation, which is a suggestive of decrease in chronic inflammatory changes and improved Nitric oxide (NO) functioning. ${ }^{21}$

\section{Haematocrit}

None of the drug is found to be statistically efficient in decreasing Haematocrit values, with $\mathrm{p}=0.973$ after intervention.

Fig 2 Effect of drug on BP, Serum Cholesterol, Triglycerides, LDL, HDL\& MDA in Atherosclerosis risk subjects (Mean \pm SEM)

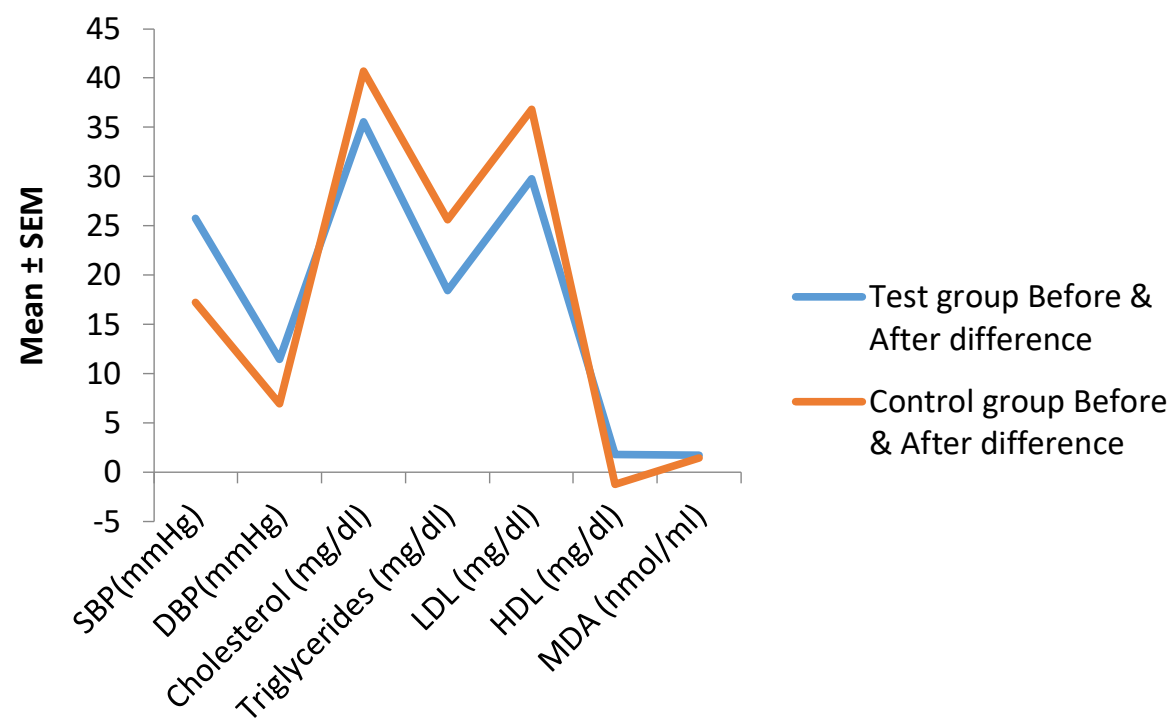

\section{Safety Parameters}

No adverse effects were observed during and after the study in both the groups. However, the Mean \pm SEM score of blood urea in test group was $26.90 \pm 7.29$ on day 0 that increased to $30.99 \pm 7.15$ on day $60^{\text {th }}, p$ $=0.032$. Clinically no adverse reaction was observed but statistically significant increment was shown in blood urea values after intervention. Cocoon of Bombyx mori is mainly comprised of protein (12-16\%). The probable reason for the increased blood urea might be due to the high protein intake, particularly sulfur containing amino acids (Methionine and Cystine) that increase endogenous acid production, causing a parallel increase in ammonia excretion. ${ }^{22}$

Findings of our study showed significant reduction in breathlessness, palpitation, chest pain, blood pressure, serum cholesterol, LDL, MDA concentration on $60^{\text {th }}$ day as compared to 0 day in test group. The pathogenesis of atherosclerosis involves two process viz oxidative stress and formation of atherosclerotic plaque. Previous studies showed that Bombyx mori(B.mori) possess antioxidant, anti-arrhythmic, antithrombotic, anti inflammatory, cholesterol-lowering, antihypertensive and anti-platelet activities in animal 
models. These activities are mainly contributed to the essential fatty acids, flavonoids, and essential amino acids present in B.mori. The essential fatty acid, $\alpha$-linolenic acid (omega-3 fatty acid), possess antiarrhythmic, anti-thrombotic and anti inflammatory activities. Through an inefficient enzymatic process of desaturation (the rate of conversion is less than 1 percent), Alpha linolenic acid (ALA) produces eicosapentaenoic acid (20 carbons) and docosahexaenoic acid (22 carbons), precursors to a group of eicosanoids (prostaglandins, thromboxanes, and leukotrienes) that are anti-inflammatory, antithrombotic, anti-arrhythmic, and vasodilator. ${ }^{23}$

The flavonoids found in the cocoon of $B$. mori comprised of three quercetin glycosides. These quercetin compounds shown to have potent anti oxidant activity as they reduce lipid peroxidation by scavenging free radicals. A part from anti oxidant, these quercetins have anti-inflammatory, anti atherogenic, anti coagulation and anti hypertensive effects too. ${ }^{24,25}$ These compounds also inhibit smooth muscle cells proliferation, migration and improve vascular function. Flavonoids reduce lipid peroxidation by scavenging ROS, chelation of transition metal ions along with reducing macrophage oxidative stress by inhibition of cellular oxygenases or by activating cellular antioxidants such as glutathione system. ${ }^{26}$

Various pharmacological studies isolated 17 amino acids from the cocoon of B. mori including histidine, lysine, aspartic acid, arginine, glutamic acid, threonine, cystine, proline, tyrosine, tryptophan, valine, phenylalanine, leucine, isoleucine, glycine, alanine and serine but majority of the amino acids are comprised of Glycine, Alanine and Serine (82\%). ${ }^{27,28}$ They aid different biological processes and played a versatile role in the prevention of atherosclerosis. Amino acids are the inhibitors of lipid peroxidation, scavengers of free radicals and chelate the transition metal ions. Sometimes they indirectly help in the reduction of ROS by assisting other anti oxidants like glutathione (an endogenous anti oxidant) that regulates Nitric oxide cycle and reduces ROS. Hence, protects against free radical mediated oxidative damage. ${ }^{29}$ Glycine has been found to exert anti inflammatory, immunomodulatory, cytoprotective, platelet stabilising and antiangiogenic effects in rodent studies. Recent studies demonstrated that pre administration of glycine reduces the infarct size by $21 \%$, which increases ventricular ejection fraction and fractional shortening. It increase endothelial Nitric oxide generation coupled with a decrease in superoxide production. Glycine exerts an anti inflammatory effect on coronary arterial cells exposed to tumour necrosis factor- $\alpha$ in vitro. ${ }^{30}$ Alanine, another important amino acid, is a precursor for the synthesis of the dipeptide- carnosine. Carnosine also has a substantial antioxidant activity as it is an electron donor, preventing lipid peroxidation and interacts with superoxide to stabilize it, chelate copper and iron and prevents the production of hydroxyl radicals. Carnosine copper complexes possess superoxide dismutase activity. Furthermore carnosine binds covalently to reactive degradation products of peroxidised lipids, thus prevent them from reacting with other cellular targets. ${ }^{31}$ Serine participates in the biosynthesis of another amino acid- Cystine. Methionine and Cystine have shown a strong positive effect on scavenging of 2, 2-diphenyl-1-picrylhydrazyl (DPPH) and hydrogen peroxide $\left.\mathrm{H}_{2} \mathrm{O}_{2}\right)$ in vitro. ${ }^{32}$ Cystine, Methionine, Glutamic acid and Glycine are required for the generation of glutathione. In cholesterol metabolism, Hydroxymethyl glutaryl co-enzyme A (HMG-CoA) reductase is activated by Glutathione. Cholesterol synthesis is suppressed by higher hepatic Glutathione content. These amino acids therefore, lowers the cholesterol. ${ }^{33}$ Another essential amino acid found in B.mori is Arginine. It also contributes to the production of Nitric oxide (natural vasodilator that helps to maintain normal blood flow in the vessels). Thus reduces blood pressure. ${ }^{34}$ Hursen, $M$ in his study found that Arginine reduces cholesterol level in blood without effecting HDL. ${ }^{35}$ Carnitine (dipeptide made of lysine and methionine) transports long chain fatty acids to mitochondria where they are consumed for energy production and lowers the cholesterol in blood. ${ }^{30}$ Taurine (organic compound formed by methionine and cystine) is important to heart muscles. The heart rhythm is regulated by potassium and calcium. They cause the tension and relaxation of the heart musculature. Taurine regulates the supply of potassium and calcium to the heart and can thereby eliminate cardiac arrhythmias. Furthermore, taurine lowers the cholesterol level as it stimulates the flow of bile. Apart from this, it also inhibits the clumping of the blood platelets. ${ }^{36,37}$ Taurine acts on nerves and reduces the signaling that decrease the resistance to blood flow and lowers the BP. ${ }^{38}$ It is found experimentally that B.mori also possess vascular anti-oxidant enzymes - SOD (Superoxide dismutases), Catalase and glutathione peroxidases. SOD, dismutate $\mathrm{O}_{2}^{\bullet-}$ to $\mathrm{H}_{2} \mathrm{O}_{2}$ and oxygen. Catalase, catalyzes the decomposition of $\mathrm{H}_{2} \mathrm{O}_{2}$ to water and oxygen. Glutathione peroxidases utilize glutathione to reduce $\mathrm{H}_{2} \mathrm{O}_{2}$ and fatty acyl peroxides to water and lipid alcohols respectively. ${ }^{39}$ 
According to Unani medicine atherosclerosis is a type of Marz-e- majari(disease of vessels) in which Sharain (arteries) become narrow or obstructed by sudda(obstruction). It is caused by raddi akhlat(morbid matter) produced as a defect of hazme saani (vascular digestion). These raddi akhlat can be akhlat-eGhaleeza (Vicid Humor) or akhlat-e- Luzuja, (Sticky Humor). As a result of which the vessels supplying the respective organs (like heart, brain, kidney etc.) also get affected and produces symptom. ${ }^{6}$ For the prevention and treatment of sudda in majari (atherosclerosis), the eminent Unani physician-Avicenna advised Istifragh-i- madda i.e. excretion of matter causing obstruction. The obstruction can be evacuated by two means-one by making the ghaleez/thick akhlat thin and other by making lesdar and luzuj/viscous matter less viscous i.e. reducing its viscosity. For the release of akhlat-e- ghaleeza(Vicid Humor) - Mulattifat (demulscent), Muhallilat(resolvent), \& Mufatteh sudud (Deobstruent) drugs are to be used that resolves thick humors into thin so that the raddi akhlat, producing obstruction can be easily washed out from the arteries. For lesdar and luzuj/viscous matter, the viscosity can be reduced by the use of Jali(detergent) and Muqatt-e-at, that not only liquefies the madda ( decreasing its viscosity) but also breaks them into small pieces so that it can be easily expel out of the vessels. ${ }^{40}$ In Tibbe Unani Aabresham(B.mori) is reported as Mulattif(demulscent), Muhallil(resolvent), Mufattite sudud(deobstruent), Muqatteh, Musaffie dam(blood purifier), Munaqqie balgham wa sauda( Expulsion of phlegm and black bile), Muqawwie jigger wa maida (Stomachic and liver tonic),Mufarrah wa Muqawwie qalb, rooh and azae raeesa (Exhilarant \& toni(c for heart and other vital organs).,8,9,10,11 $\operatorname{Aabresham(B.mori)}$ by virtue of its resolving and demulscent properties resolve thick and viscous humors. It's Muqatteh, deobstruent and blood purifying effect breaks akhlate luzuja (Plaques forming matter) in small pieces which are then evacuated from the vessels by its munaqqie balgham and sauda property. Thus, facilitates smooth flow of humors, that strengthen and tafreeh the qalb, rooh, and azae raeesa(exhilarate and strengthens the heart and other vital organs). Its Muqawwie maida wa jigger (Stomachic and liver tonic) activities strengthen and improve the quwwate tabiyah(natural faculties) thereby produces the akhlate saleh i.e. reduces the total cholesterol. Hence, the mechanism proposed by the Unani physicians appears to be comprehensive and very much in proportion with the modern approach of prevention.

The above discussion pointed Aabresham(B.mori) as a promising anti-atherogenic and cardio protective drug still needs further evaluation on large sample size.

\section{Control group}

Atorvastatin was used as a standard control drug in this study. Atorvastatin is a member of well known drugStatins, used for lowering blood cholesterol that works by inhibiting Hydroxymethyl glutaryl co-enzyme A (HMG-CoA) reductase, an enzyme found in liver tissue that plays a key role in the production of cholesterol in body. It also acts as anti inflammatory thereby stabilizes the plaque and prevents stroke. ${ }^{41}$ Control group equally exhibited significant reduction in palpitation, breathlessness, headache, chest pain, blood pressure, total serum cholesterol, LDL, and MDA concentration after intervention as do the test group.

\section{Conclusion:}

The overall effect of Aabresham (B.mori) was found quite encouraging in the atherosclerosis risk group. The efficacy of Aabresham (B.mori) was observed equal to the standard drug i.e. Atorvastatin in reducing palpitation, breathlessness, chest pain, blood pressure, total serum cholesterol, LDL and MDA concentration. The findings outlined above are due to the potent antioxidant, anti inflammatory, anti arrhythmic, anti angiogenic, anti thrombotic, anti coagulant, anti hypertensive, cholesterol and LDL lowering effects of Aabresham(B.mori). All these effects illustrate that the use of Aabresham(B.mori) potentially inhibit the development and rate of progression of atherosclerosis. It is suggestive to intercalate the treatment modalities like weight management, dietary modification and increased physical activity along with Aabresham (B.mori).

\section{References:}

1. W. Slijkhuis, W. Mali, Y. Appelman. A historical perspective towards a non-invasive treatment for patients with atherosclerosis. Netherlands Heart Journal. 2009 April; 17(4): p. 140-141. 
2. Anonymous. Atherosclerosis.National Heart,Lung and Blood Institute. [Online]; 2016 Jan. Available from: http://www.nhlbi.nih.gov/health-topics/atherosclerosis/

3. Shanthi Mendis, Pekka Puska, Bo Norrving. Global Atlas on cardiovascular disease prevention and control. World Health Organization. Geneva; 2011: p. 1-153.

4. World Health Organization. Cardiovascular diseases. [Online] Geneva 2011. Available from: http://sancd.org/uploads/pdf/factsheet_CHD.

5. Jane A. Leopold, Joseph Loscalzo. Oxidative Risk for Atherothrombotic Cardiovascular Disease. Free Radic Biol Med. 2009 December 15; 12(47): p. 1673-1706.

6. Rushd I. Kitabul Kulliyat. New Delhi: CCRUM; 1989: p. 87-90.

7. Ghani N. Khazainul Advia. New Delhi: Idara Kitabul Shifa; 2010: p. 749-750.

8. Anonymous. High blood cholesterol.ATP III Guidelines at a glance quick desk reference. National heart,lung and blood institute; 2001.

9. Gururaj M. Kuppuswamy's Socio-Economic Status Scale - A Revision of Income Parameter For 2014. International Journal of Recent Trends in Science And Technology. 2014; 11(1): p. 1-2.

10. The Criteria Committee for the New York Heart Association. Nomenclature and Criteria for Diagnosis of Diseases of the Heart and Great Vessels. 9th ed: Little Brown and Company; 1994.

11. Michael S, Peter J I. congestive heart failure: Diagnosis,Pathophysiology, Therapy and implementations of Respiratory care. Respiratory Care. 2006 April; 51(4): p. 403-410.

12. J. C. Witteman, D. E. Grobbee, F. J. Kok, A. Hofman, and H. A. Valkenburg.Increased risk of atheroscle rosis in women after the menopause. BMJ. 1989 March; 298(6674): p.642-644.

13. Manish Verma, Poonam Verma, Shabnam Parveen, Karuna Dubey. Comparative Study of Lipid Profile Levels in Vegetarian and Non-Vegetarian Person. International Journal of Life-Sciences Scientific Research. 2015 Nov; 1( 2): p: 89-93

14. George Howard,etal.Cigarette Smoking and Progression of Atherosclerosis. The Atherosclerosis Risk in Communities (ARIC) Study. JAMA. 1998 ; 279(2) : p.119-124.

15. Panniyammakal Jeemon, K.S. Reddy. Social determinants of cardiovascular disease outcomes in Indians. Indian J Med Res. 2010 Nov; 132(5): p. 617-622.

16. Pandey AK, Pandey S, Blaha MJ, Agatston A, Feldman T, Ozner M , et al. Family history of coronary heart disease and the incidence and progression of coronary artery calcification: Multi-Ethnic Study of Atherosclerosis (MESA). Elsevier. 2013 March; 232 (2014): p. 369-376.

17. Yu Chen, Wade K Copeland, Rajesh Vedanthan, Eric Grant, Jung Eun Lee,Dongfeng Gu, etal.Associati on between body mass index andcardiovascular disease mortality in east Asiansand south Asians: pooled analysis of prospectivedata from the Asia Cohort Consortium. BMJ . 2013 Oct; 347: p. 1-25.

18. World Health Organization. Physical inactivity a leading cause of disease and disability, warns.WHO.Geneva.[Online];2016.Availablefrom:http://www.who.int/mediacentre/news/release23/en/

19. Georgia V, Dimitris T, Christodoulos S. The Role of Oxidative Stress in Atherosclerosis. Hellenic J Cardiol. 2009;(50): p. 402-409.

20. David Weiner, William E.Mitch, Jeff M.Sands. Clinical journal of the American society of nephrology. [Online]; 2014. Available from: http://cjasn.asnjournals.org/content/early/

21. Maggie B C. Omega3 Fatty Acids. American family physicians. 2014 July 1; 1(70): p. 133-144.

22. Islam M. An update on the cadiovascular effects of qercetin, a plant flavonoid. Current nutrition and food science. 2014;(10): p. 36-48.

23. Gross M. Flavonoids and Cardiovascular Disease. Pharmaceutical Biology. 2004; 42: p. 21-35.

24. M. Mondal, K.Trivedy,S.Nirmal K. The silk proteins, sericin and fibroin in silkworm, Bombyx mori Linn. - a review. Caspian Journal of Environmental Sciences. 2007; 5(2): p. 63-76.

25. Kushal Sen MB. Studies on Indian silk.I.Macrocharacterization and analysis of amino acid composition. Journal of applied polymer science. 2004 Feb 18; 92(2): p. 1080-1097.

26. Guiseppe cirillo Fl. Anti oxidant polymer synthesis,properties and application: Wiley; 2012: p. 12-37.

27. Mark F Mc Carty, James J Di Nicolantonio. The cardiometabolic benefits of glyceine:Is glycine an antidote to dietary fructose. Open Heart. 2014;1(1): p.1-9.

28. Mark F Mc Carty, James J Di Nicolantonio. $\beta$-Alanine and orotate as supplements for cardiac protection.Open Heart. 2014;1(1): p.1-9. 
29. Aluko Chibuike, C. Udenigwe, Rotimi E. Chemometric Analysis of the Amino Acid Requirements of Antioxidant Food Protein Hydrolysates. International Journal of Molecular Sciences. 2011; 12: p. 3148 3161.

30. Oda H. Functions of Sulfur-Containing Amino Acids in Lipid Metabolism. The journal of nutrition. 2006: p. 1666-1669.

31. David Gersten.Amino Acids and Heart Health. The Life Connection. [Online]; 2013. Available from: http://lifeconnectionmagazine.com/amino-acids-and-heart-health-by-david-gersten-med/

32. Hursen M, Regan MC, Krik SJ. Metabolic effects of arginine in a healthy elderly population. Journal of parenteral and enteral nutrition. 1995; 19: p. 227-230.

33. K, Sumizu. Oxidation of hypotaurine in rat liver. Biochim. Biophys. Acta. 1962; 63: p. 210

34. Chapman RA, Suleinan MS, Earm YE. Taurine and the heart. Cardiovascular research.1993; 27(3): p. 358-363.

35. Ibn Sina. Al Qanoon (Urdu translation by Kantoori GH). New Delhi:Idara Kitabul Shifa; 2007: p. 349,758-766.

36. David Gersten.Amino Acids and Heart Health. The Life Connection. [Online]; 2013. Available from: http://lifeconnectionmagazine.com/amino-acids-and-heart-health-by-david-gersten-med/

37. Hursen M, Regan MC, Krik SJ. Metabolic effects of arginine in a healthy elderly population. Journal of parenteral and enteral nutrition. 1995; 19: p. 227-230.

38. K, Sumizu. Oxidation of hypotaurine in rat liver. Biochim. Biophys. Acta. 1962; 63: p. 210

39. Chapman RA, Suleinan MS, Earm YE. Taurine and the heart. Cardiovascular research.1993; 27(3): p. 358-363.

40. Ibn Sina. Al Qanoon (Urdu translation by Kantoori GH). New Delhi:Idara Kitabul Shifa; 2007: p. 349,758-766.

41. Wikipedia.Atorvastatin.[Online];2014 Feb.Available from: http://Atorvastatin\%20-\%20Wikipedia,\%20the\%20free\%20encyclopedia.html 